\title{
Severe bilateral adrenal hemorrhages in a newborn complicated by persistent adrenal insufficiency
}

\author{
Nicholas R Zessis', Jennifer L Nicholas² and Stephen I Stone1 \\ 'Pediatrics and 2Radiology, Washington University in St. Louis School of Medicine, St. Louis, Missouri, USA
}

Correspondence

should be addressed

to SI Stone

Email

sstone@wustl.edu

\section{Summary}

Bilateral adrenal hemorrhages rarely occur during the neonatal period and are often associated with traumatic vaginal deliveries. However, the adrenal gland has highly regenerative capabilities and adrenal insufficiency typically resolves over time. We evaluated a newborn female after experiencing fetal macrosomia and a traumatic vaginal delivery. She developed acidosis and acute renal injury. Large adrenal hemorrhages were noted bilaterally on ultrasound, and she was diagnosed with adrenal insufficiency based on characteristic electrolyte changes and a low cortisol (4.2 $\mathrm{mg} / \mathrm{dL})$. On follow-up testing, this patient was unable to be weaned off of hydrocortisone or fludrocortisone despite resolution of hemorrhages on ultrasound. Providers should consider bilateral adrenal hemorrhage when evaluating critically ill neonates after a traumatic delivery. In extreme cases, this may be a persistent process.

\section{Learning points:}

- Risk factors for adrenal hemorrhage include fetal macrosomia, traumatic vaginal delivery and critical acidemia.

- Signs of adrenal hemorrhage include jaundice, flank mass, skin discoloration or scrotal hematoma.

- Adrenal insufficiency often is a transient process when related to adrenal hemorrhage.

- Severe adrenal hemorrhages can occur in the absence of symptoms.

- Though rare, persistent adrenal insufficiency may occur in extremely severe cases of bilateral adrenal hemorrhage.

- Consider adrenal hemorrhage when evaluating a neonate for shock in the absence of an infectious etiology.

\section{Background}

Adrenal hematoma is the most common adrenal mass in newborns. Adrenal hemorrhage (AH) of the newborn has a wide spectrum of disease severity and, as a result, the literature reports a varying incidence, initially thought to range from 1.7 to 3 per 1000 live births (1). Majority of AH is, however, asymptomatic and the frequency at which it occurs incidentally has only recently been appreciated (1). Several studies retrospectively assessed the presence of AH with screening abdominal ultrasounds in newborns, suggesting a more common occurrence of 16-29 per 1000 live births (2). Most newborns with recognized disease do not require treatment, as even 10\% functional cortisolproducing tissue is sufficient to prevent adrenal insufficiency (AI) (3). Additionally, the newborn adrenal is a highly regenerative organ. Those that do progress to $\mathrm{AI}$ and require treatment, though rare, show quick resolution (1). We offer a literature review of $\mathrm{AH}$ in newborns, as well as a unique presentation of bilateral disease in the setting of traumatic delivery. Our patient has been followed to three years of age and has shown persistent primary AI. Such a severe case, to the best of our knowledge, has not been documented in the literature with $\mathrm{AH}$ as the primary etiology. 


\section{Case presentation}

Our female patient was born to a 29-year-old mother at an estimated 38 4/7-week gestational age. The pregnancy was complicated by gestational hypertension, and no history of diabetes mellitus. Her delivery was complicated by prolonged rupture of membranes $(20 \mathrm{~h})$ and nonreassuring fetal status. Most significantly, there was nearly six minutes of shoulder dystocia, ultimately requiring an emergent episiotomy. Her resuscitation was significant for initial pulselessness with chest compressions for the first two minutes of life. Apgar scores were 0, 2, 5, 6 and 8 at $0,1,5,10$ and $15 \mathrm{~min}$ of life, respectively. Birth weight was $4250 \mathrm{~g}$ (98th percentile), length was $53 \mathrm{~cm}$ (97th percentile) and occipitofrontal circumference was $36.8 \mathrm{~cm}$ (99th percentile). This was consistent with large for gestational age and macrosomia. She was hypotensive, without abdominal masses or splenomegaly. Skin showed no bruising or petechiae. She was afebrile and initial laboratory studies pointed against an infectious process. Her right extremity examination was concerning for brachial plexus injury. Her neurologic examination, as well as her EEG, were consistent with neonatal encephalopathy.

\section{Investigation}

Her early course was complicated by mild rhabdomyolysis secondary to her traumatic delivery. Additionally, she had a metabolic acidosis $\left(\mathrm{pH} 7.16 / \mathrm{pCO}_{2} 56 / \mathrm{HCO}_{3}{ }^{-} 20 /\right.$ base excess -9), indicating poor end-organ perfusion. Transaminases were elevated, consistent with acute liver injury. Finally, she featured an elevated creatinine, azotemia and oliguria consistent with an acute impairment of renal perfusion. This prompted a renal ultrasound, which revealed extremely large bilateral AHs (Fig. 1). Early labs featured characteristic signs of hypoadrenalism including hyponatremia, hyperkalemia, metabolic acidosis and low random cortisol in the setting of a critical illness (Table 1). Infectious and coagulation studies were normal.

\section{Treatment}

The patient was started on stress dose hydrocortisone $\left(50 \mathrm{mg} / \mathrm{m}^{2} /\right.$ day divided three times daily) on day of life (DOL) two and fludrocortisone on DOL three (initially $0.2 \mathrm{mg}$ daily, then weaned to $0.1 \mathrm{mg}$ daily the following day). Within $48 \mathrm{~h}$, she had rapid correction of hypotension and electrolyte abnormalities and was weaned to hydrocortisone dose of $15 \mathrm{mg} / \mathrm{m}^{2} /$ day. Our group has been following this patient with serial measurement of electrolytes, adrenocorticotropic hormone (ACTH) and plasma renin activity while on therapy (Fig. 2). The patient required sodium chloride supplementation (2g daily) until she was able to regulate her salt intake with table foods at 18 months. She was maintained on fludrocortisone $0.1 \mathrm{mg}$ daily.

\section{Outcome and follow-up}

She failed a high-dose $(250 \mu \mathrm{g})$ ACTH stimulation test at age 13 months (labs drawn fasting at 08:00 h). Her baseline ACTH level was elevated $(159 \mathrm{pg} / \mathrm{mL})$.

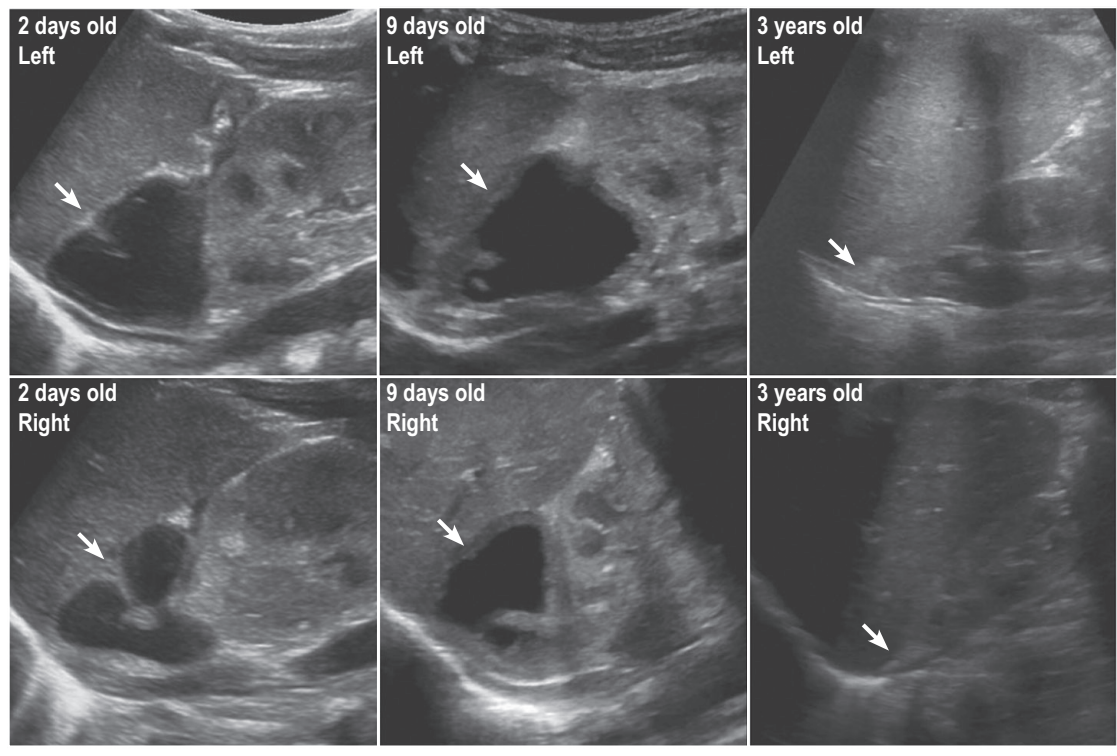

\section{Figure 1}

Grayscale ultrasound images of the bilateral adrenal fossa in the long axis. Large cystic mass noted in the center of the adrenals bilaterally consistent with adrenal hemorrhages. This is evolving in the early neonatal period. By 3 years of age, these lesions have resolved without adrenal calcification. 
Table 1 Representative neonatal laboratory studies. As a neonate, the patient experienced several laboratory derangements. This included metabolic acidosis, anemia, thrombocytopenia, hyponatremia, hyperkalemia, azotemia, rhabdomyolysis and elevated transaminases. The low random cortisol in the setting of characteristic electrolyte changes and critical illness was concerning for primary adrenal insufficiency.

\begin{tabular}{lcc}
\hline Parameters & Values \\
\cline { 1 - 2 } $\mathrm{pCO}_{2}(\mathrm{mmHg})$ & 7.16 \\
$\mathrm{HCO}_{3}(\mathrm{mmol} / \mathrm{L})$ & 56 \\
$\mathrm{WBC}(\mathrm{K} / \mathrm{cumm})$ & 13 \\
$\mathrm{Hgb}(\mathrm{g} / \mathrm{dL})$ & 12.7 \\
$\mathrm{Plt}(\mathrm{K} / \mathrm{cumm})$ & 10.4 \\
$\mathrm{Na}(\mathrm{mmol} / \mathrm{L})$ & 86 \\
$\mathrm{~K}(\mathrm{mmol} / \mathrm{L})$ & 121 \\
$\mathrm{Cl}(\mathrm{mmol} / \mathrm{L})$ & 5.4 \\
$\mathrm{BUN}(\mathrm{mg} / \mathrm{dL})$ & 92 \\
$\mathrm{Cr}(\mathrm{mg} / \mathrm{dL})$ & 41 \\
$\mathrm{AST}(\mathrm{U} / \mathrm{L})$ & 4.4 \\
$\mathrm{ALT}(\mathrm{U} / \mathrm{L})$ & 771 \\
$\mathrm{CK}(\mathrm{U} / \mathrm{L})$ & 545 \\
$\mathrm{Cortisol}(\mu \mathrm{g} / \mathrm{dL})$ & 6017 \\
$\mathrm{PT}(\mathrm{s})$ & 4.2 \\
$\mathrm{PTT}(\mathrm{s})$ & 19 \\
\hline
\end{tabular}

ALT, alanine transaminase; AST, aspartate transaminase; BUN, blood urea nitrogen; $\mathrm{CK}$, creatine kinase; $\mathrm{Cl}$, serum chloride; cortisol, random cortisol; $\mathrm{Cr}$, serum creatinine; $\mathrm{HCO}_{3}{ }^{-}$, serum bicarbonate; $\mathrm{Hgb}$, hemoglobin; $\mathrm{K}$, serum potassium; $\mathrm{Na}$, serum sodium; $\mathrm{pCO}_{2}$, arterial partial pressure of carbon dioxide; $\mathrm{pH}$, arterial potential of hydrogen; Plt, platelet; PT, prothrombin time; PTT, partial thromboplastin time; WBC, white blood cell count.

Cortisol levels were 3.5, 5.1 and 5.2 at 0,30 and $60 \mathrm{~min}$ respectively. This suggests insufficient residual cortisolsecreting tissue. Renal ultrasound at three years of age showed resolution of $\mathrm{AH}$ without evidence of calcification (Fig. 1). The patient recovered from her neonatal encephalopathy and has a very mild palsy of her right upper extremity.

\section{Discussion}

$\mathrm{AH}$ can be classified into an acquired, traumatic or idiopathic etiology. Acquired causes, usually seen outside of the newborn period, include WaterhouseFriderichsen syndrome, primary antiphospholipid syndrome and anticoagulation (4). There are several risk factors that have been linked to $\mathrm{AH}$, many of which predispose the newborn to a traumatic vaginal delivery. Diabetic mothers, large-for-gestational-age infants, perinatal asphyxia, breech delivery, forceps or vacuum delivery, sepsis, thrombocytopenia, hypothrombinemia, disseminated intravascular coagulation, Factor V Leiden and prolonged labor are the major risk factors $(1,4,5)$. The largest study with a cohort of neonatal $\mathrm{AH}$ found that the two most common variables are fetal acidemia (31\%) and macrosomia (22\%). Interestingly, AH has increased in frequency despite falling rates of vaginal deliveries over the last two decades, likely attributable to the increasing incidence of macrosomia (2).

There is a wide-ranging spectrum of disease. $90 \%$ of cases were initially believed to be unilateral, with $75 \%$ right sided $(3,4)$. The vast majority do not result in impaired adrenal function. Calcified central hematomas of the adrenal are incidentally identified by radiograph or autopsy in older infants and children, suggesting not all $\mathrm{AH}$ are immediately fatal (4). Presentation is delayed, in part, to the adrenal gland's strong regenerative capacity (5).

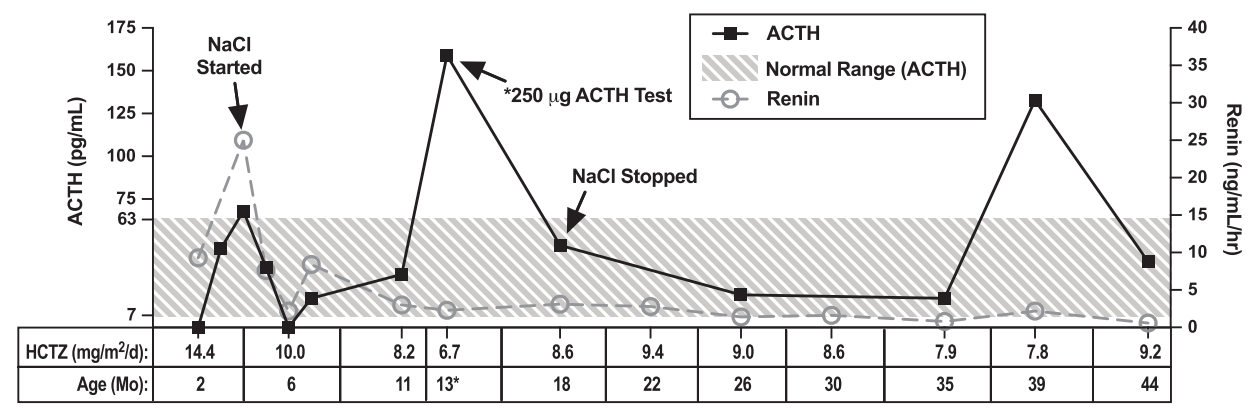

Figure 2

Serial laboratory studies and medication management. After being on a relatively high dose of hydrocortisone (HCTZ), the patient's hydrocortisone replacement was weaned to $8-10 \mathrm{mg} / \mathrm{m}^{2} /$ day. This resulted in stable adrenocorticotropic hormone (ACTH) levels over time. *The patient was intentionally weaned to a subphysiologic dose of hydrocortisone at 13 months in preparation for a high-dose ACTH test. On $6.7 \mathrm{mg} / \mathrm{m}^{2} / \mathrm{day}$, her baseline ACTH spiked to $159 \mathrm{pg} / \mathrm{mL}$. At 39 months of age as the patient outgrew her hydrocortisone dose $\left(7.8 \mathrm{mg} / \mathrm{m}^{2} / \mathrm{day}\right)$, she again had an elevated ACTH of $132.7 \mathrm{pg} / \mathrm{mL}$. These spikes in ACTH when the patient is on subphysiologic hydrocortisone replacement are suggestive of permanent primary adrenal insufficiency. The patient's fludrocortisone requirements have remained stable ( $0.1 \mathrm{mg}$ daily). The patient did require sodium chloride (NaCl) supplementation between 4 and 18 months. After 18 months, the patient was able to regulate her salt intake in her diet and her plasma renin activity has remained normal. 
The presence of minimal functioning residual tissue is enough to prevent AI. Even with bilateral hemorrhage, both glands are unlikely to be affected to the same extent (6). Most hemorrhage is subcapsular, sparing the glucocorticoid-producing cortex, further explaining the rarity of Addisonian crisis (3). One study followed 37 affected newborns to the resolution of hemorrhage, finding a range of 3-9 months (7). In a different study, the mean duration for resolution is about 18 weeks (2).

Several hypotheses have been proposed regarding the pathophysiology of AH. As the injury is evolving, the venous sinusoids of the adrenal cortex become distended with blood. This results in increased pressure, decreased blood flow and tissue hypoxia (1, 3, 5). A single adrenal vein drains each adrenal. Under stress, with elevated ACTH, arterial blood flow is stimulated and can cause adrenal vein spasm so that the limited venous drainage capacity may cause venous stasis and venous thrombosis, leading to hemorrhage (2). In the setting of shock, there may be reflex shunting away from splanchnic circulation, leading to infarction (1). A study assessing the histology of 122 affected newborns showed changes consistent with infarction, suggesting hypoxic-ischemic events with subsequent reperfusion injury as the culprit (6).

In studying newborns of vaginal deliveries, microscopic hemorrhage in the cortex at birth is very common even in the absence of risk factors. More significant $\mathrm{AH}$, however, likely develops within the first $24 \mathrm{~h}$ of life (8). The predilection for right-sided hemorrhage is likely from compression of the adrenal gland between the liver and spine. With the right adrenal vein draining into the inferior vena cava, compression is likely to induce venous pressure changes (6).

The symptoms and signs of AH in newborns are varied, with the most severe cases resulting in profound shock with AI, often with cyanosis $(3,4)$. This includes jaundice (seen in up to $50 \%$ of cases), flank mass, overlying skin discoloration and scrotal hematoma in males $(2,3,4,7)$. Laboratory changes expected in the setting of AI include anemia, hyponatremia, hyperkalemia, hypoglycemia, hypocortisolemia, eosinophilia, leukocytosis, elevated adrenocorticotropic hormone and elevated renin activity $(4,8)$. Infants have a relatively greater requirement for aldosterone than do older children, owing to renal immaturity, as well as the low sodium content of breast milk and formula. Therefore, hyperkalemia, hyponatremia and hypoglycemia are prominent presenting signs in infants (4). Newborns with AI will become ill on the order of days secondary to electrolyte disturbances. The most definitive study is a high-dose ACTH stimulation test (4), although false negatives for AI may occur if performed close to the hemorrhage insult, as there may still be functional glucocorticoid-producing tissue. In instances in which the diagnosis is not apparent, unexplained anemia, persistent indirect hyperbilirubinemia and abdominal distension may be clues to investigate for AH. In these instances, there is still potential to escalate to hypovolemic shock and AI (2). Ultrasound is the recommended imaging study to assess for $\mathrm{AH}(4,8)$. Radiographs may identify rim-like calcifications as early as the fifth DOL, but this typically occurs over weeks (5).

There are several comorbidities to consider in the newborn with AH. Poor cortisol production increases the risk for neonatal hypoglycemia, especially in newborns of diabetic mothers. Cortisol deficiency may alter hepatic metabolism of catecholamines, leading to neonatal cholestasis (1). Rupture of the adrenal capsule can lead to intraperitoneal leakage, often resulting in scrotal hematoma (6). Under a similar process, intestinal obstruction may occur with rupture or mass effect secondary to hematoma (5).

The literature offers diverse presentations of $\mathrm{AH}$. Velaphi and coworkers describe a newborn with bilateral involvement that presented with hypotension, however, passed an ACTH stimulation test on DOL five (6). Klemm and coworkers discuss several newborns presenting with circulatory collapse and major salt loss in the setting of $\mathrm{AH}$, one of which failed an ACTH stimulation test initially, but passed at 2 months of age (9). Morgan and coworkers present a newborn with congenital heart disease with persistent hypotension, found to be cortisol deficient. Both glucocorticoid and mineralocorticoid replacement was necessary, though by DOL 74, only fludrocortisone was needed (10). The most extensive study (2) involved over 26000 newborns screened with abdominal ultrasound. $0.28 \%$ were found to have $\mathrm{AH}$ with a male-to-female ratio of 1.55:1.71 of 74 cases were vaginal deliveries, and there was no significant difference between right- and left-sided hemorrhage when unilateral, in stark contrast to the studies preceding it. Of note, only one of the 74 babies had bilateral involvement and did develop AI.

We present a novel case of permanent acquired AI diagnosed in the neonatal period. After a severely traumatic delivery, our patient presented with signs of poor end-organ perfusion. She had characteristic laboratory findings of Addison's disease, such as hyponatremia and hyperkalemia, as described in Saevik and coworkers (11). Macrosomia and fetal acidemia were her known risk factors. Though she presented in shock with characteristic salt loss, she did not have the typical examination signs characteristic 
of AH. Sonographic evidence of severe AH was evident in the newborn period. She is the exception to the rule with regards to the regenerative capacity of the adrenal gland, as her AI has proven to be unremitting. She continues to require exogenous glucocorticoid and mineralocorticoid through 3 years of age, indicative of primary disease.

We encourage clinicians to consider $\mathrm{AH}$ when evaluating a neonate for shock in the absence of an infectious etiology. This is especially prudent as the incidence of $\mathrm{AH}$ is more common than previously recognized. However, relatively few patients progress to AI and only in extreme presentations, does it persist into childhood.

\section{Declaration of interest}

The authors declare that there is no conflict of interest that could be perceived as prejudicing the impartiality of the reported research.

\section{Funding}

This research did not receive any specific grant from any funding agency in the public, commercial, or not-for-profit sector.

\section{Patient consent}

The parents of the child described in the manuscript have agreed to allow for publication of the child's medical history. Every effort has been taken to keep her protected health care information anonymous.

\section{Author contribution statement}

$\mathrm{N} \mathrm{Z}$ and S S contributed to the conception and design of this manuscript. $\mathrm{J} \mathrm{N}$ assisted with analysis and interpretation of the adrenal ultrasound imaging.

\section{Acknowledgements}

The authors would like to thank the child described in this manuscript as well as her parents for agreeing to share her medical history. The authors would also like to acknowledge Fumihiko Urano for his critical review and feedback with this manuscript.

\section{References}

1 Koklu E, Kurtoglu S, Akcakus M \& Koklu S. Adrenal haemorrhage with cholestasis and adrenal crisis in a newborn of a diabetic mother. Journal of Pediatric Endocrinology and Metabolism 200720 441-444. (https://doi.org/10.1515/jpem.2007.20.3.441)

2 Gyurkovits Z, Maróti Á, Rénes L, Németh G, Pál A \& Orvos H. Adrenal haemorrhage in term neonates: a retrospective study from the period 2001-2013. Journal of Maternal-Fetal and Neonatal Medicine 201528 2062-2065. (https://doi.org/10.3109/14767058.20 14.976550)

3 Ruffini E, De Petris L, Zorzi G, Paoletti P, Mambelli G \& Carlucci A. Two cases of neonatal adrenal hemorrhage presenting with persistent jaundice. La Pediatria Medica e Chirurgica 201335 285-287. (https:// doi.org/10.4081/pmc.2013.26)

4 White PC. Adrenocortical insufficiency. In Nelson Textbook of Pediatrics, 20th ed., ch. 2703-2714. Eds RM Kliegman, BF Stanton, JWS Geme, NF Schor \& RE Behrman. Philadephia, PA: Elsevier, 2016.

5 Hawdon J, Cheetham T, Schenk DJ, Wraith JE, Jones SA \& Bishop N. Metabolic and endocrine disorders. In Rennie and Robertson's Textbook of Neonatology, 5th ed. Ed JM Rennie. London, UK: Elsevier Limited, 2012.

6 Velaphi SC \& Perlman JM. Neonatal adrenal hemorrhage: clinical and abdominal sonographic findings. Clinical Pediatrics $2001 \mathbf{4 0}$ 545-548. (https://doi.org/10.1177/000992280104001002)

7 Demirel N, Bas AY, Zenciroglu A \& Tasci-Yildiz Y. Adrenal bleeding in neonates: report of 37 cases. Turkish Journal of Pediatrics $20115 \mathbf{5 3}$ $43-47$.

8 Jacobsson H, Kaiser S, Granholm T \& Ringertz HG. Neonatal adrenal haemorrhage at bone scintigraphy: a case report. Pediatric Radiology 199828 896-898. (https://doi.org/10.1007/s002470050492)

9 Klemm W, Bierich JR, Moller H \& Nolte K. Acute adrenal hemorrhage in newborn infants: the importance of early diagnosis. Monatsschrift Kinderheilkunde $1976 \mathbf{1 2 4} 106-109$.

10 Morgan LM, Austin EH \& Holland BJ. Large bilateral adrenal haemorrhages in a newborn with unrepaired cyanotic CHD. Cardiology in the Young 201626 1005-1007. (https://doi.org/10.1017/ S1047951116000263)

11 Sævik ÅB, Åkerman AK, Grønning K, Nermoen I, Valland SF Finnes TE, Isaksson M, Dahlqvist $\mathrm{P}$, Bergthorsdottir R, Ekwall $\mathrm{O}$, et al. Clues for early detection of autoimmune Addison's disease - myths and realities. Journal of Internal Medicine 2017283 190-199. (https:// doi.org/10.1111/joim.12699)

Received in final form 14 January 2018

Accepted 23 January 2018 